\title{
Novel Test of Modified Newtonian Dynamics with Gas Rich Galaxies
}

\begin{abstract}
Stacy S. McGaugh
Department of Astronomy, University of Maryland, College Park, Maryland 20742-2421, USA

(Received 12 November 2010; revised manuscript received 20 January 2011; published 21 March 2011)

The current cosmological paradigm, the cold dark matter model with a cosmological constant, requires that the mass-energy of the Universe be dominated by invisible components: dark matter and dark energy. An alternative to these dark components is that the law of gravity be modified on the relevant scales. A test of these ideas is provided by the baryonic Tully-Fisher relation (BTFR), an empirical relation between the observed mass of a galaxy and its rotation velocity. Here, I report a test using gas rich galaxies for which both axes of the BTFR can be measured independently of the theories being tested and without the systematic uncertainty in stellar mass that affects the same test with star dominated spirals. The data fall precisely where predicted a priori by the modified Newtonian dynamics. The scatter in the BTFR is attributable entirely to observational uncertainty, consistent with a single effective force law.
\end{abstract}

The mass discrepancy problem in extragalactic systems is well established. When known dynamical laws are applied to these systems, the observed mass in stars and gas falls well short of explaining the observed motions. A classic example is that the rotation curves of disk galaxies tend to become roughly flat $\left(V_{f} \sim\right.$ const) when they should be falling in a Keplerian $\left(V \propto r^{-1 / 2}\right)$ fashion. The common interpretation for this phenomenon is dark matter. However, a logical alternative is that the dynamical laws that lead to the inference of dark matter need to be revised on the scales appropriate to galaxies.

One striking fact about extragalactic systems is that they are many orders of magnitude larger than the solar system in which conventional dynamics is extraordinarily well tested. One idea is thus to modify gravity on some suitably large length scale such that the apparent need for dark matter would be manifest in galaxies but not in the solar system. Such size-dependent ideas fail and can generically be excluded [1]. However, there are other ways in which galaxies differ from the solar system. For example, the centripetal acceleration required to keep a star in orbit in a galaxy is very much lower than that experienced by the planets orbiting the sun: $\sim 10^{-10} \mathrm{~ms}^{-2}$ vs $6 \times 10^{-3} \mathrm{~ms}^{-2}$ for Earth.

The modified Newtonian dynamics (MOND) [2] posits a new constant with dimensions of acceleration, $a_{0}$, which defines the boundary between conventional dynamics and a new domain of dynamics. The conventional dynamics hold in the limit of high acceleration, $a \gg a_{0}$, and the modified regime occurs in the limit of low accelerations, $a \ll a_{0}$. The value of $a_{0}$ must be determined observationally [3], but once specified is constant. In the modified regime, rotation curves become asymptotically flat far from a central mass [2]. This follows from the scale invariance symmetry of the equations of motion under transformations $(t, r) \rightarrow(\lambda t, \lambda r)$ [4]. An absolute relation between the asymptotically flat rotation velocity $V_{f}$ and the total mass $M_{b}$,

$$
a_{0} G M_{b}=V_{f}^{4},
$$

follows uniquely on dimensional grounds.

Rotationally supported galaxies follow an empirical relation between mass and rotation velocity known as the baryonic Tully-Fisher relation (BTFR) [5]. This empirical relation can, in principle, provide a quantitative test of the prediction of MOND. In order to do so, we require independent, accurate measurements of both $M_{b}$ and $V_{f}$. While the latter is readily obtained from resolved rotation curves, mass determinations are more problematic.

The baryonic mass is the sum of both stars and gas: $M_{b}=M_{\star}+M_{g}$. A great deal is known about stars, but stellar mass estimates for galaxies are subject to a systematic uncertainty of $\sim 0.15$ dex because of uncertainty in the stellar mass function and some details of stellar evolution [6]. This level of systematic uncertainty precludes an unambiguous test of (1) with star dominated spirals [7].

A clean test of the BTFR predicted by MOND follows if we can identify a class of galaxies where stars do not dominate the baryonic mass budget. Atomic gas typically dominates the mass of nonstellar material in disk galaxies. Its mass follows directly from the distance to each galaxy, the measured $21 \mathrm{~cm}$ flux, and the physics of the spin flip transition of hydrogen. It does not suffer from the systematic uncertainty of stellar mass.

Late type, low surface brightness disk galaxies frequently have gas masses in excess of their stellar masses [8]. When $M_{g}>M_{\star}$, the systematic uncertainty in stellar mass is reduced to a minor contributor to the error budget (Fig. 1). Thanks to recent work [9-11], it is now possible to assemble a large sample (47) of galaxies with $V_{f}$ measured from resolved rotation curves that satisfy the gas domination criterion $M_{g}>M_{\star}$. This property enables a novel test of MOND with no free parameters. Both $M_{b}$ and $V_{f}$ are directly measured and, for the first time, are not dominated by systematic uncertainties. Moreover, these 

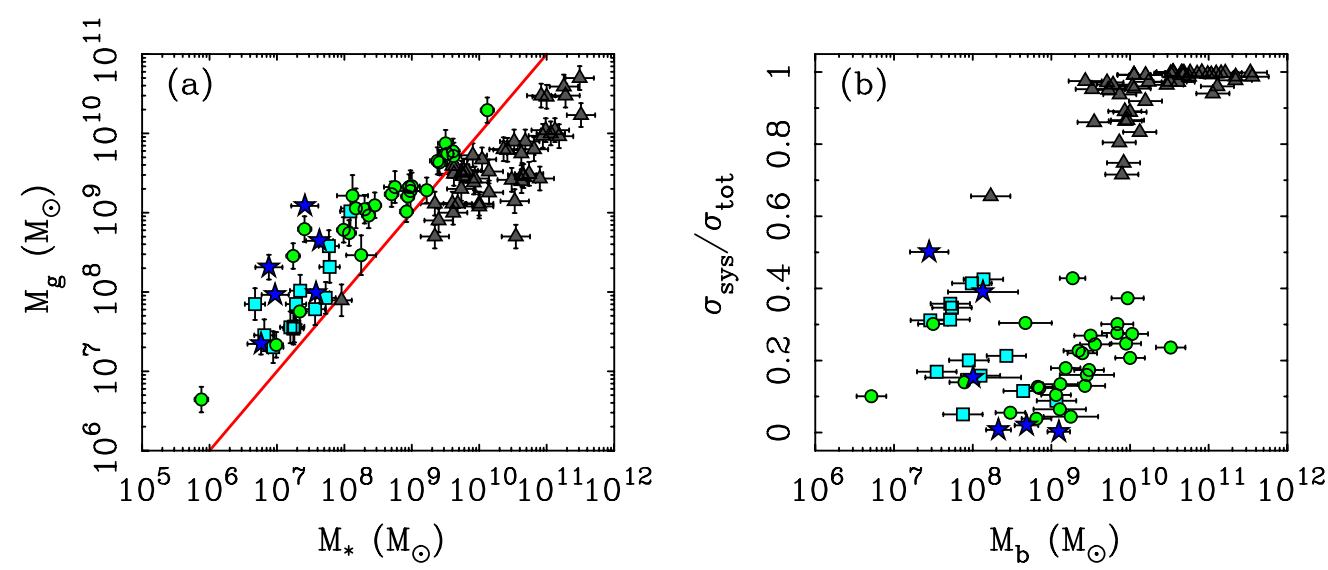

FIG. 1 (color online). (a) The mass of stars and gas in rotating galaxies. Triangles represent star dominated spirals [7] with $M_{\star}>$ $M_{g}$. The data for gas rich galaxies with $M_{\star}<M_{g}$ come from several independent sources denoted by circles [9], squares [10], and stars [11]. (b) The fraction of the error budget $\sigma_{\text {sys }} / \sigma_{\text {tot }}$ contributed by the systematic uncertainty in stellar mass $\left(\sigma_{\text {sys }} \approx 0.15\right.$ dex [6]) as a function of the total baryonic mass $M_{b}=M_{\star}+M_{g}$. Our knowledge of the masses of star dominated galaxies is limited by this systematic uncertainty, but it has little effect on gas dominated galaxies.

galaxies are unambiguously in the deep modified regime where (1) holds, with $V_{f}^{2} / r_{\max } \approx a_{0} / 10$. Here the distinction between MOND and $\Lambda \mathrm{CDM}$ is most pronounced.

Figure 2 shows the gas rich galaxy data together with the predictions of MOND and the cold dark matter model with a cosmological constant $(\Lambda \mathrm{CDM})$. The data fall precisely where MOND predicts. This happens with no fitting whatsoever-there are zero free parameters in Fig. 2. Computing $\chi^{2}$ with the slope fixed to 4 and the normalization fixed at the previously determined value of $a_{0}$ [3] gives $\chi^{2}=44.3$ for 46 degrees of freedom for a reduced $\chi_{\nu}^{2}=0.96$. If we treat $a_{0}$ as a fit parameter and minimize

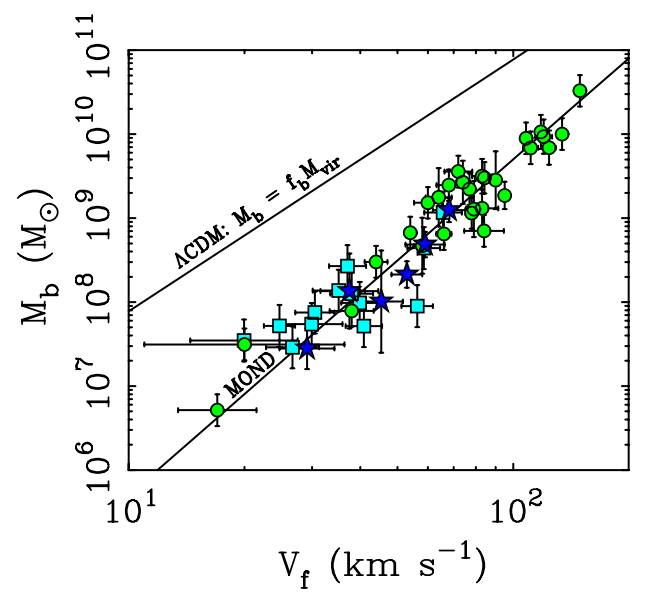

FIG. 2 (color online). The BTFR for gas dominated galaxies. The sum of detected baryonic mass, stars and gas, is plotted against the flat rotation velocity $V_{f}$ (symbols as per Fig. 1). Both mass and velocity are measured independently of either MOND or $\Lambda \mathrm{CDM}$. The data are well removed from the expectation of the standard cosmology (upper line), but follow the prediction of MOND (lower line) with no fitting whatsoever. $\chi^{2}$ with the slope fixed to 4 , we find $a_{0}=1.24 \pm 0.14 \times$ $10^{-10} \mathrm{~m} \mathrm{~s}^{-2}$. This is indistinguishable from the previous value, and $\chi^{2}$ actually increases because we have added an unneeded degree of freedom: $\chi_{\nu}^{2}=0.99$. If we further treat the slope as an additional free parameter, we find $3.8 \pm 0.2$. This does not differ significantly from the MOND prediction of 4 , nor does it improve the fit: $\chi_{\nu}^{2}=$ 0.98 . The data therefore provide no reason to suspect a BTFR that differs in any way from that predicted by MOND.

The specific BTFR that the data follow is unique to MOND. Indeed, to the best of my knowledge, MOND is the only theory to make a strong a priori prediction for the BTFR. The dark matter paradigm makes no comparably iron-clad prediction.

The expectation in $\Lambda \mathrm{CDM}$ is that total mass (both dark and baryonic) scales with rotation velocity as $M_{\Delta}=$ $(\Delta / 2)^{-1 / 2}\left(G H_{0}\right)^{-1} V_{\Delta}^{3}$. These quantities are defined at a radius where the enclosed density exceeds the cosmic critical density by a factor $\Delta$. The virial radius occurs at $\Delta \approx 100$ [12]. This notional radius is well beyond the reach of observations. To plot the $\Lambda \mathrm{CDM}$ line in Fig. 2, we assume $V_{f}=V_{\text {vir }}$ and $M_{b}=f_{b} M_{\text {vir }}$ where $f_{b}=0.17$ is the cosmic baryon fraction [13]. This nominal expectation has the wrong slope and the wrong normalization.

In order to reconcile $\Lambda \mathrm{CDM}$ with the data, we must invoke additional parameters. The simplest assumption is that only a fraction $f_{d}$ of the baryons in a halo are detected: $M_{b}=f_{d} f_{b} M_{\mathrm{vir}}$. Once we have granted ourselves this freedom, a galaxy could, in principle, have any $f_{d}<1$ and reside anywhere below the $\Lambda \mathrm{CDM}$ line in Fig. 2. From this perspective, it is puzzling that galaxies reside only along the line predicted by MOND.

Reproducing the observed BTFR in $\Lambda \mathrm{CDM}$ requires a remarkable degree of fine-tuning. The detected baryon 
fraction must follow the formula $\log f_{d}=$ $\log \left(V_{f} / 100 \mathrm{~km} \mathrm{~s}^{-1}\right)-1.2$. Astrophysical feedback is often invoked to cause $f_{d}<1$, but provides no satisfactory explanation for why this particular tuning of $f_{d}$ arises.

A further test is provided by the scatter in the observed relation. In $\Lambda \mathrm{CDM}$, any scatter in $f_{d}$ translates directly into the BTFR: there should be at least some intrinsic scatter. In MOND, the BTFR is a consequence of the force law, and should have no intrinsic scatter.

Figure 3 shows a histogram of the ratio a $=V_{f}^{4} /\left(G M_{b}\right)$ formed from the data. This represents the scatter around the MOND line in Fig. 2. If the data are randomly distributed, they should approximate a gaussian whose width is dictated by the size of the error bars. Such a Gaussian is shown in Fig. 3. It is not fit to the data; it is simply centered on the previously determined value of $a_{0}$ [3] with a width corresponding to the uncertainty in the data. Only random errors are considered here; the residual systematic uncertainty in the stellar mass corresponds to a small shift in the total mass and should not introduce additional scatter.

Observational uncertainty suffices to explain the scatter in the data. The data are consistent with a BTFR of zero intrinsic width. This is natural if the BTFR is imposed by the force law, as in MOND. It is not expected in $\Lambda$ CDM where there should be many sources of scatter.

From the perspective of cosmology, it is disturbing that MOND works at all. If $\Lambda \mathrm{CDM}$ is the correct paradigm, this should not happen [14]. Yet when pressed into a new regime where the predictions of the two theories are distinct, MOND outperforms $\Lambda$ CDM.

This is not the first time that strong predictions of MOND have been realized. For example, MOND predicted in advance that galaxies of both high and low surface

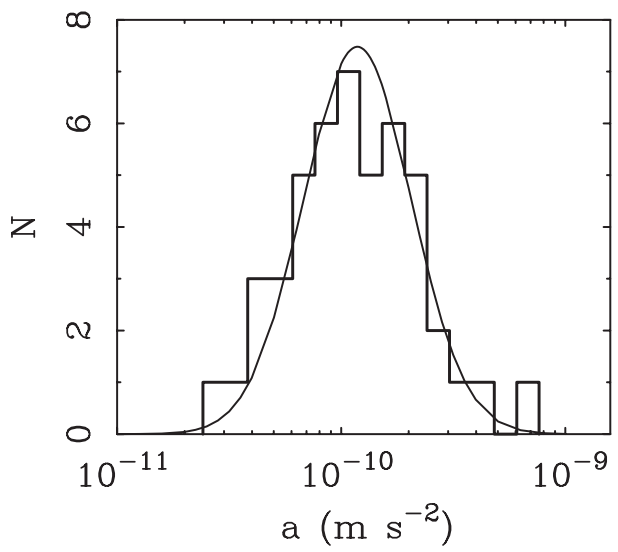

FIG. 3. Histogram of the measured values a $=V_{f}^{4} /\left(G M_{b}\right)$. The data are consistent with a normal distribution (smooth curve) that is centered on the previous determination of $a_{0}$ [3] with a width specified by the mean uncertainty $\sigma=0.24$ dex. This consistency implies a universal acceleration scale with negligible intrinsic scatter. This is expected in MOND, but poses a finetuning problem for $\Lambda \mathrm{CDM}$. brightness would fall on the same BTFR [15,16], contrary to the natural expectation of purely Newtonian gravity $[17,18]$. It is well established that MOND provides good fits to the detailed shapes of rotation curves with only the stellar mass-to-light ratio as a free parameter [19]. The required mass-to-light ratios are in good agreement with stellar population synthesis models [20]. A simple model motivated by MOND provided the only successful a priori prediction of the first-to-second peak amplitude ratio of the acoustic peaks of the cosmic background radiation: $A_{1: 2}=2.4$ predicted [21] vs $2.34 \pm 0.09$ measured [22]. It is rare for a noncanonical theory to have so many predictive successes.

MOND also has its share of problems. The same ansatz that correctly predicted the second acoustic peak amplitude also predicts a lower third peak than is observed [23]. This does not falsify MOND, but it does imply that a generally covariant parent theory should provide an effective forcing term [24].

The most serious observational problem facing MOND is the dynamics of rich clusters of galaxies. These appear to weigh more than can be accounted for with the observed baryons even with the modified dynamics $[25,26]$. This residual mass discrepancy is roughly a factor of 2 in mass. On the one hand, this is very disturbing - a theory that seeks to eliminate the need for cosmic dark matter itself suffers a missing mass problem. On the other hand, this is less severe than the missing baryon problem in $\Lambda \mathrm{CDM}$, where dwarf galaxies are missing $99 \%$ of the baryons that should be associated with their dark matter halos [27]. So both theories suffer a missing baryon problem, albeit of different amplitudes in systems of vastly different scale.

While some of the mass in clusters appears to be dark, even in MOND, there is nothing that requires this unseen mass to be in some new form of nonbaryonic particle. Indeed, big bang nucleosynthesis implies the existence of considerably more baryons than have so far been detected [28]. If only a fraction of these missing baryons reside in clusters it would suffice to resolve the residual mass discrepancy suffered by MOND.

Perhaps the most prominent example of a cluster with a serious residual discrepancy in MOND is the bullet cluster [29]. In this system, the gravitational lensing of background galaxies indicates that the mass is offset from the $\mathrm{X}$-ray plasma. This is the same residual mass discrepancy that is seen in all rich clusters. While the bullet cluster is frequently cited as evidence against MOND, it is also problematic for $\Lambda \mathrm{CDM}$. The subclusters that compose the bullet cluster collided at a remarkably high velocity $\left(\sim 4700 \mathrm{~km} \mathrm{~s}^{-1}\right)$. This is exceedingly unlikely in $\Lambda \mathrm{CDM}$, occurring with a probability of only a few parts in a billion [30]. In contrast, such high collision velocities are natural to MOND [31]. Taken at face value, the bullet cluster would seem to simultaneously support and falsify both theories with equal vigor. 
Given the nature of astronomical data, some exceptions to any theory are to be expected. What is surprising in the case of MOND is that it continues to enjoy predictive successes at all. These motivate the search for a more complete gravitational theory that contains MOND in the appropriate limit [32-35].

It is possible that nonbaryonic cold dark matter does not exist. If it does, and $\Lambda \mathrm{CDM}$ is the correct solution, the challenge is to understand the empirical systematics encapsulated in the simple MOND formula. These are not native to the current cosmological paradigm [14] but must be explained by any successful theory.

Another possibility is that dark matter particles have properties that impose MOND-like phenomenology [36-38]. In this case, it is desirable to have dark matter that behaves like standard cold dark matter on large scales, but which interacts with normal matter so as to impose the MOND phenomenology in galaxies. This suggests some strong new form of interaction between dark matter and baryons.

If MOND is essentially correct in that it is pointing towards an extension of gravitational theory, then the experiments seeking to detect dark matter will find null results. This would also be the case if dark matter has a different nature than currently presumed. If dark matter is detected, then the MOND formula is still useful as a phenomenological constraint on the effective force law in spiral galaxies. In any case, the predictive power of the simple formula proposed by Milgrom [2] is telling us something profound about nature.

I thank Benoit Famaey for organizing the conference where these issues came into focus. I also thank Moti Milgrom and Bob Sanders for sharing their perspectives, and the referees for their constructive comments. The work of SSM is supported in part by NSF grant AST0908370.

[1] S. S. McGaugh and W. J. G. de Blok, Astrophys. J. 499, 41 (1998).

[2] M. Milgrom, Astrophys. J. 270, 365 (1983).

[3] The value $a_{0}=1.21 \pm 0.24 \times 10^{-10} \mathrm{~ms}^{-2}$ was obtained by K. G. Begeman, A. H. Broeils, and R. H. Sanders, Mon. Not. R. Astron. Soc. 249, 523 (1991) from detailed fits to the rotation curves of star dominated galaxies. Their procedure necessarily treats the mass-to-light ratio of each galaxy as a free parameter. The use of gas rich galaxies here allows us to avoid this additional freedom.

[4] M. Milgrom, Astrophys. J. 698, 1630 (2009).

[5] S.S. McGaugh, J.M. Schombert, G.D. Bothun, and W. J. G. de Blok, Astrophys. J. 533, L99 (2000).

[6] C. Conroy, J. E. Gunn, and M. White, Astrophys. J. 699, 486 (2009).
[7] S. S. McGaugh, Astrophys. J. 632, 859 (2005).

[8] J. M. Schombert, S. S. McGaugh, and J. A. Eder, Astron. J. 121, 2420 (2001).

[9] D. V. Stark, S. S. McGaugh, and R. A. Swaters, Astron. J. 138, 392 (2009).

[10] A. Begum, J. N. Chengalur, I. D. Karachentsev, and M. E. Sharina, Mon. Not. R. Astron. Soc. 386, 138 (2008).

[11] C. Trachternach, W. J. G. de Blok, S. S. McGaugh, J. M. van der Hulst, and R. Dettmar, Astron. Astrophys. 505, 577 (2009).

[12] H. J. Mo and S. Mao, Mon. Not. R. Astron. Soc. 353, 829 (2004). Numerically, the proportionality constant is $4.6 \times$ $10^{5} \mathrm{M}_{\odot} \mathrm{km}^{-3} \mathrm{~s}^{3}$ for $H_{0}=72 \mathrm{~km} \mathrm{~s}^{-1} \mathrm{Mpc}^{-1}$.

[13] E. Komatsu et al., Astrophys. J. Suppl. Ser. 180, 330 (2009).

[14] R.H. Sanders, Adv. Astron. Astrophys. 2009, 752439 (2009).

[15] M. A. Zwaan, J. M. van der Hulst, W. J. G. de Blok, and S. S. McGaugh, Mon. Not. R. Astron. Soc. 273, L35 (1995).

[16] D. Sprayberry, G. M. Bernstein, C. D. Impey, and G. D. Bothun, Astrophys. J. 438, 72 (1995).

[17] S. S. McGaugh and W. J. G. de Blok, Astrophys. J. 499, 66 (1998).

[18] S. S. McGaugh, Phys. Rev. Lett. 95, 171302 (2005).

[19] R.H. Sanders and S.S. McGaugh, Annu. Rev. Astron. Astrophys. 40, 263 (2002).

[20] A. Hasani Zonoozi and H. Haghi, Astron. Astrophys. 524, A53 (2010).

[21] S. S. McGaugh, Astrophys. J. 523, L99 (1999).

[22] L. Page et al., Astrophys. J. Suppl. Ser. 148, 39 (2003).

[23] S. S. McGaugh, Astrophys. J. 611, 26 (2004).

[24] C. Skordis, D. F. Mota, P. G. Ferreira, and C. Boehm, Phys. Rev. Lett. 96, 011301 (2006).

[25] R. H. Sanders, Mon. Not. R. Astron. Soc. 342, 901 (2003).

[26] G. W. Angus, B. Famaey, and D. A. Buote, Mon. Not. R. Astron. Soc. 387, 1470 (2008).

[27] S. S. McGaugh, J. M. Schombert, W. J. G. de Blok, and M. J. Zagursky, Astrophys. J. 708, L14 (2010).

[28] M. Fukugita, C. J. Hogan, and P. J. E. Peebles, Astrophys. J. 503, 518 (1998).

[29] D. Clowe, A. Gonzalez, and M. Markevitch, Astrophys. J. 604, 596 (2004).

[30] J. Lee and E. Komatsu, Astrophys. J. 718, 60 (2010).

[31] G. W. Angus and S. S. McGaugh, Mon. Not. R. Astron. Soc. 383, 417 (2007).

[32] J. D. Bekenstein, Phys. Rev. D 70, 083509 (2004).

[33] J. R. Brownstein and J. W. Moffat, Astrophys. J. 636, 721 (2006).

[34] M. Milgrom, Phys. Rev. D 80, 123536 (2009).

[35] P.D. Mannheim and J.G. O'Brien, arXiv:1007.0970 [Phys. Rev. Lett. (to be published)].

[36] L. Blanchet, Classical Quantum Gravity 24, 3529 (2007).

[37] J. Bruneton, S. Liberati, L. Sindoni, and B. Famaey, J. Cosmol. Astropart. Phys. 03 (2009) 021.

[38] H. Zhao and B. Li, Astrophys. J. 712, 130 (2010). 